\title{
P03-5-4 Poster session
}

\section{Protective effect of artesunate and aspirin in experimental model of colorectal cancer}

\author{
Sneh Verma ${ }^{1}$, Prasenjit Das ${ }^{2}$, Vijay L. Kumar ${ }^{1}$ \\ ${ }^{1}$ Department of Pharmacology, All India Institute of Medical Sciences, New Delhi, India, ${ }^{2}$ Department of Pathology, All \\ India Institute of Medical Sciences, New Delhi, India
}

Background The risk reduction of colorectal cancer with anti-inflammatory agents mainly aspirin is now very well established. Artesunate, an anti-malarial drug has a plethora of pharmacological activities including anti-inflammatory, gastro-protective and anti-colitic. In view of this, the present study was designed to evaluate the protective effect of artesunate in an experimental model of colorectal cancer in rat and to compare it with that of aspirin.

Methods The study was carried out in male Wistar rats where pre-cancerous changes like multiple plaque lesions (MPL) and aberrant crypt foci (ACF) in the colon were induced by two injections of 1,2-dimethylhydrazine ( $\mathrm{DMH}_{3} 150 \mathrm{mg} / \mathrm{kg}$ each) administered subcutaneously at an interval of 1 week. The effects of artesunate (50 and $150 \mathrm{mg} / \mathrm{kg})$ and aspirin (60 $\mathrm{mg} / \mathrm{kg}$ ) at their anti-inflammatory doses were studied after 8 weeks of 2 nd injection of $\mathrm{DMH}_{\text {s }}$ the time at which ACF count was found to be at its peak. Parameters like MPL and ACF count and crypt multiplicity along with histoarchitecture of colonic mucosa was evaluated. The protective effect of drugs was also evaluated on markers of Wnt/ $\beta$-catenin signaling, angiogenesis, cellular proliferation and apoptosis.

Results Both artesunate and aspirin significantly reduced MPL and ACF count, crypt multiplicity and restored colonic histoarchitecture in DMH treated rat. These changes were accompanied by significant reduction in the levels/expression of $\beta$-catenin, VEGF, MMP-9, PCNA and Ki67 as compared to DMH treated control group. Treatment with artesunate and aspirin also enhanced DNA fragmentation, TUNEL positivity and Bax/Bcl2 immunoreactivity.

Conclusion The protective effect of artesunate and aspirin was attributed to down-regulation of $\beta$-catenin, suppression of angiogenesis, cellular proliferation and induction of apoptosis. The findings of the study suggest that like aspirin, use of artesunate could decrease the risk of colon cancer. 